\title{
Effect of the Enrichment Medium on the Detection and Diversity of Salmonella from Porcine Duodenal Content
}

\author{
Emily V. De Busser, Dominiek Maes, Kurt Houf, Jeroen Dewulf, and Lieven De Zutter
}

\begin{abstract}
This study assesses the effect of the enrichment medium used on the isolation of Salmonella from the duodenal content of naturally infected slaughter pigs. At six slaughterhouses, the duodenum was collected from 458 randomly chosen pigs and examined in the laboratory. Three semi-solid enrichment media (modified semisolid Rappaport-Vassiliadis medium [MSRV], diagnostic semi-solid Salmonella medium [DIASALM], and Simple Method Salmonella [SMS] agar) and three enrichment broths (Rappaport-Vassiliadis, Rappaport Vassiliadis broth with Soya [RVS], and Muller Kauffmann Tetrathionate novobiocin broth [MKTTn]) were evaluated. If a migration zone was present on the semi-solid media, a loopful was taken both near the inoculation drop and at the edge of the migration zone and streaked on a Xylose Lysine Desoxycholate (XLD) agar plate. Each enrichment broth was streaked on XLD, and three presumptive colonies were further examined. Detection rate was calculated, and isolates were, after serotyping, genotyped by performing pulsed-field gel electrophoresis (PFGE). The overall frequency of Salmonella isolated in at least one of the six different media was $15.5 \%(71 / 458)$. No significant differences in relative sensitivity were obtained within semi-solid media and within liquid media. Semi-solid media showed a significant higher relative sensitivity than the one obtained with liquid media. A relative sensitivity higher than $83.1 \%$, namely of $94.4 \%$, could only be obtained by combining three different enrichment media (MSRV or DIASALM+RVS+MKTTn). In $13.4 \%$ of the positive pigs, more than one serotype was found within the duodenum of one pig. In $12.9 \%$ of the duodenal contents, different genotypes were found within the same serotype. Differences in serotypes and genotypes were found predominantly within the same enrichment medium. In conclusion, to obtain the highest Salmonella detection rate in naturally contaminated pig samples, MSRV should be used as enrichment medium. However, to obtain a realistic picture of the sero- and genotypes present, different samples per enrichment medium and different enrichment media should be tested.
\end{abstract}

\section{Introduction}

$\mathbf{E}$ UROPEAN UNION (EU) DIRECTIVES foresee reduction targets for Salmonella in food and animal populations as part of the overall EU strategy to reduce foodborne diseases in humans (Regulation [EC] No 2160/2003). In this context, several surveys obtaining reliable and comparable data on the Salmonella prevalence in pigs in EU Member States have already been carried out (Hald et al., 2003; EFSA, 2008, 2010a). Further, national monitoring programs have been established in which bacteriological isolation forms an important part. To obtain comparable results from different countries, it is essential that Salmonella isolation procedures are performed accurately and in a standardized manner.

Different media and culture methods are available for the isolation of Salmonella. The detection of Salmonella spp. in animal feces and in samples from primary production is described in ISO 6579:2002/Amd 1 2007, in which the selective enrichment medium used is the modified semi-solid Rappaport-Vassiliadis (MSRV), developed for the detection of motile Salmonella (EFSA, 2010b) and in which subculture is carried out from the migrated culture, with the inoculum taken from the edge of the visual growth zone. Non-motile Salmonella are therefore not isolated. As only Salmonella Gallinarum and Salmonella Pullorum are not motile (May and Goodner, 1927) and are host specific (poultry), the use of MSRV should not lead to false negative results in Salmonella isolation from porcine samples. However, not all salmonellae have the same capacity to migrate on the medium and some may have evolved into non-motile variants (Grimont et al., 2000).

Previous research has shown that the semi-solid media diagnostic semisolid Salmonella medium (DIASALM) and

Faculty of Veterinary Medicine, Ghent University, Merelbeke, Belgium. 
MSRV are not suitable for all Salmonella serotypes (O'Donoghue and Winn, 1993; Read et al., 1994). A combination of one of these with the liquid medium Rappaport-Vassiliadis (RV) leads to a higher detection rate of Salmonella (Voogt et al., 2001). A further complication is that multiple serotypes can be present in samples from naturally infected animals (Funk et al., 2000; O'Carroll et al., 1999), raising the question whether all of these have an equal chance of being detected (Singer et al., 2009). Probably a particular serotype grows over the others during incubation. This selection pressure has already been described by Harvey and Price's (1967) demonstrating that different Salmonella serotypes had different growth characteristics in the same selective enrichment broths. However, Singer et al. (2009) suggest that the factors influencing this in vitro variability are not solely due to growth competition among Salmonella serotypes, as inconsistent results were also found in a fecal experiment tube containing a single Salmonella strain.

Further questions involve the number of samples which should be taken and how many suspected colonies derived from the enrichment medium should be tested. These often depend on the objective of the study (e.g., to indicate the samples as Salmonella positive or negative, to study the epidemiology of Salmonella or to investigate the origin of contaminated food involved in an outbreak). As in the latter two, identifying the sero- and genotypes present is of major importance.

In this study, the impact of six different enrichment media on the detection rate and diversity of Salmonella from duodenal content of slaughter pigs is examined.

\section{Materials and Methods}

\section{Samples}

The study was conducted from December 2006 to August 2007. A total of 458 pigs were randomly selected at six different slaughterhouses (A-F). In total, 22 slaughterhouse visits were performed (2-10 visits per slaughterhouse; 10-28 pigs per visit) and 56 different slaughter batches were included. (A slaughter batch contains pigs originating from the same herd.) During evisceration, stomach-gut packages were collected, the duodenum was ligated, taken out, transferred into a sterile bag, and transported to the laboratory.

\section{Salmonella isolation}

Upon arrival in the laboratory, the samples were immediately processed for Salmonella isolation. Each duodenum was immersed in 95\% ethanol and dried in air before being cut open with sterile utensils. Ten grams of duodenal content was diluted 1/10 with buffered peptone water (BPW; Bio-Rad, Marnes-La-Coquette, France), homogenized in a stomacher blender and incubated at $37^{\circ} \mathrm{C}$. After $16-20 \mathrm{~h}$, $0.1 \mathrm{~mL}$ of the BPW broth was added to $10 \mathrm{~mL}$ of RV (Oxoid Ltd., Hampshire, UK) and $10 \mathrm{~mL}$ of Rappaport Vassiliadis broth with Soya (RVS; Bio-Rad), spotted on DIASALM (Lab M Ltd., Topley House, Lancashire, UK) and Simple Method Salmonella (SMS; AES Chemunex, BruzCedex, France) agar, and dispersed in three drops on MRSV (Lab M Ltd. Topley House). Subsequently, $1 \mathrm{~mL}$ of the BPW culture was also added to $10 \mathrm{~mL}$ of Muller Kauffmann Tetrathionate novo- biocin broth (MKTTn; Oxoid Ltd.). After incubation of the enrichment media for $24 \mathrm{~h}$ at $42^{\circ} \mathrm{C}$ (but $37^{\circ} \mathrm{C}$ for MKTTn), the DIASALM, SMS, and MSRV plates were examined for the presence of typical migration zones, and a loopful of the migration zone near the inoculation drop and also from the edge of the migration zone was streaked on a Xylose Lysine Desoxycholate (XLD; Bio-Rad) agar plate. A loopful from each RV, RVS, and MKTTn enrichment broth was also streaked on a XLD agar plate. After incubation for $24 \mathrm{~h}$ at $37^{\circ} \mathrm{C}$, all XLD plates were examined for the presence of typical colonies, ranging from pink colonies with large, glossy black centers to almost completely black ones. From the semi-solid agars and the enrichment broths, one and three typical colonies, respectively, per XLD plate were selected for identification. In this way, a maximum of $15 \mathrm{col}-$ onies per duodenal sample was obtained. Collected isolates were biochemically tested using triple sugar iron, indol, and lysine.

\section{Evaluation of strain motility}

In nine pigs, Salmonella could only be isolated after enrichment in one or more broths. The isolates $(n=18)$ obtained from these pigs were cultured in Trypto-Casein-Soy Broth (TSB) (Bio-Rad) for $24 \mathrm{~h}$ in $37^{\circ} \mathrm{C}$. After overnight incubation, two dilutions $\left(10^{6}\right.$ and $\left.10^{3} \mathrm{cfu} / \mathrm{mL}\right)$ were made in $0.1 \%$ peptone water. Of these dilutions, $0.1 \mathrm{~mL}$ was spotted on MSRV in three drops and incubated for $24 \mathrm{~h}$ at $42^{\circ} \mathrm{C}$. The migration capacity of the strains was evaluated by checking the presence of a migration zone on the MSRV agar after incubation. As a reference, 31 isolates obtained from the enrichment broths, but originating from a duodenal sample that tested positive on all enrichment media, were included and submitted to the above mentioned culture method.

\section{Salmonella serotyping}

To limit the number of strains that had to be serotyped, all Salmonella isolates were subjected to an enterobacterial repetitive intergenic consensus (ERIC) polymerase chain reaction (PCR) (Rasschaert et al., 2005). At least two isolates per cluster were then selected and serotyped by the Belgian reference laboratory for Salmonella (Veterinary and

Table 1. Relative Sensitivities of EnRichment Media Used for SALMONELLA ISOLATION From Duodenal Content of Slaughter Pigs (71 Items Positive in at Least One Method)

\begin{tabular}{lcc}
\hline $\begin{array}{l}\text { Enrichment } \\
\text { medium }\end{array}$ & $\begin{array}{c}\text { Number of } \\
\text { positive pigs }\end{array}$ & $\begin{array}{c}\text { Relative } \\
\text { sensitivity (\%) }\end{array}$ \\
\hline MSRV & 59 & 83.1 \\
DIASALM & 58 & 81.7 \\
SMS & 58 & 81.7 \\
RV & 40 & 56.3 \\
RVS & 39 & 54.9 \\
MKTTn & 34 & 47.9 \\
All media & 71 & 100 \\
\hline
\end{tabular}

MSRV, modified semi-solid Rappaport-Vassiliadis medium; DIASALM, diagnostic semi-solid Salmonella medium; SMS, Simple Method Salmonella; RV, Rappaport Vassiliadis; RVS, Rappaport Vassiliadis broth with Soya; MKTTn, Muller Kauffmann Tetrathionate novobiocin broth. 
Table 2. Results of McNemar Test and Level of Agreement (Kappa VAlues) for Different EnRichment Media

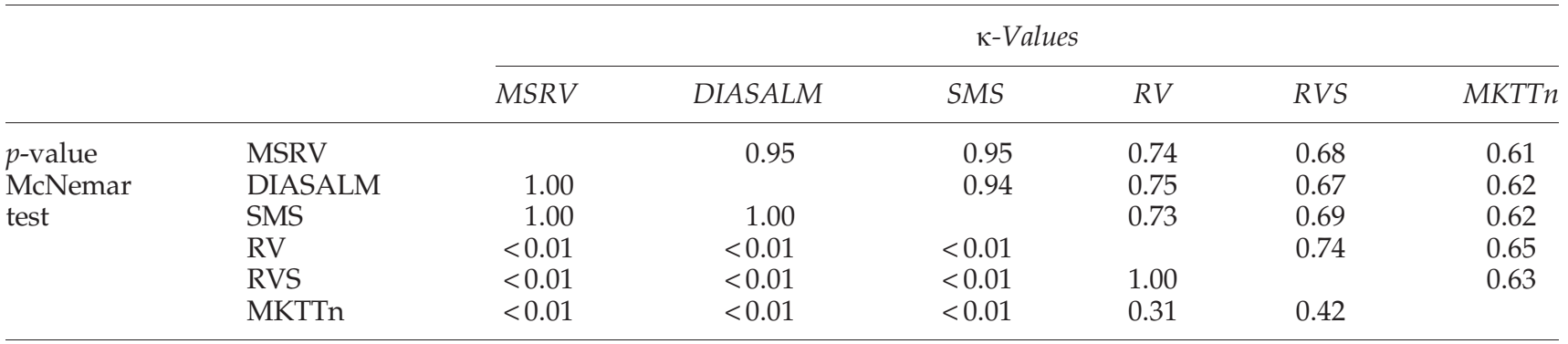

MSRV, modified semi-solid Rappaport-Vassiliadis medium; DIASALM, diagnostic semi-solid Salmonella medium; SMS, Simple Method Salmonella; RV, Rappaport Vassiliadis; RVS, Rappaport Vassiliadis broth with Soya; MKTTn, Muller Kauffmann Tetrathionate novobiocin broth.

Agrochemical Research Centre, Ukkel, Belgium) using the Kaufmann-White scheme (Popoff and Le Minor, 1992).

\section{Salmonella genotyping}

When more than one isolate with the same serotype was present in a duodenal sample, characterization on strain level was performed by pulsed-field gel electrophoresis (PFGE). The PulseNet protocol (Ribot et al., 2006) was used with the following modification. The running condition was $6 \mathrm{~V} / \mathrm{cm}$ at $14^{\circ} \mathrm{C}$ in $0.5 \times$ Tris-Borate-EDTA buffer for $20 \mathrm{~h}$ with a ramping time from 2.2 to 54.2 seconds. Profiles were obtained by GelCompar II (3.5) (Applied Maths, Sint-Martems-Latem, Belgium) using the Dice coefficient with $2.1 \%$ position tolerance, and clusters were generated through the unweighted pair group method using arithmetic averages algorithm (UPGMA). A PFGE genotype was assigned on the basis of a difference in at least one band in the $\mathrm{XbaI}$ fingerprint. Genotypes within serotypes are indicated below by the capital of the name of the serotype followed by a number (e.g., Salmonella Typhimurium genotype 1 is indicated as $\mathrm{T}_{1}$ ).

\section{Statistical analysis}

The relative sensitivity of each culture method was calculated as the number of positive duodenal samples detected by that method, divided by the total number of samples that tested positive by at least one of the six methods (gold standard).

Possible differences between the proportions of positive pigs obtained with each culture method were analyzed using McNemar tests (SPSS version 19). The level of statistical significance used was $p<0.05$.

To detect the agreement between the results of the culture methods used, kappa values were calculated (SPSS 19). Interpretation of the kappa values was made according to Landis and Koch (1977): $\leq 0$, poor agreement; 0.01-0.20, slight agreement; $0.21-0.40$, fair agreement; $0.41-0.60$, moderate agreement; $0.61-0.80$, substantial agreement; $0.81-1.00$, almost perfect agreement.

\section{Results}

In $15.5 \%(71 / 458)$ of the samples, Salmonella was isolated by at least one of the media. The relative sensitivity of each medium is shown in Table 1. No significant differences were found between the proportion of positive samples obtained by the different semi-solid media or by the different liquid media. However, the proportion of positive samples obtained by the semi-solid media significantly $(p<0.05)$ differed from those obtained by the liquid media (Table 2 ).

Table 3. Number of Pigs (with Percentages) for Specific Salmonella Serotypes Using DifFerent Enrichment Media

\begin{tabular}{|c|c|c|c|c|c|c|c|}
\hline Serotype & $M S R V$ & DIASALM & SMS & $R V$ & $R V S$ & MKTTn & $\begin{array}{c}\text { Total } \\
\text { positive pigs }\end{array}$ \\
\hline Total positive pigs & 59 & 58 & 58 & 40 & 39 & 34 & 71 \\
\hline Salmonella Typhimurium & $41(69.5 \%)$ & $41(70.7 \%)$ & $38(65.5 \%)$ & $25(62.5 \%)$ & $19(48.7 \%)$ & $21(61.8 \%)$ & 45 \\
\hline Salmonella Derby & $12(20.3 \%)$ & $12(20.7 \%)$ & $13(22.4 \%)$ & 7 (17.5\%) & $10(25.6 \%)$ & $7(20.6 \%)$ & 15 \\
\hline Salmonella Anatum & $3(5.1 \%)$ & $3(5.2 \%)$ & $3(5.2 \%)$ & $3(7.5 \%)$ & $3(7.7 \%)$ & $3(8.8 \%)$ & 3 \\
\hline Salmonella Infantis & $1(1.7 \%)$ & $1(1.7 \%)$ & $1(1.7 \%)$ & $1(2.5 \%)$ & $1(2.6 \%)$ & $1(2.9 \%)$ & 1 \\
\hline Salmonella Brandenburg & $2(3.4 \%)$ & $1(1.7 \%)$ & $1(1.7 \%)$ & $1(2.5 \%)$ & $1(2.6 \%)$ & 0 & 2 \\
\hline Salmonella Rissen & $3(5.1 \%)$ & $1(1.7 \%)$ & $2(3.4 \%)$ & $1(2.5 \%)$ & $1(2.6 \%)$ & 0 & 4 \\
\hline Salmonella O4:i:- & $1(1.7 \%)$ & 0 & 0 & $1(2.5 \%)$ & $1(2.6 \%)$ & $2(5.9 \%)$ & 3 \\
\hline Salmonella Ohio & 0 & $1(1.7 \%)$ & 0 & $1(2.5 \%)$ & $1(2.6 \%)$ & 0 & 1 \\
\hline Salmonella Arizonae & 0 & 0 & 0 & 0 & 0 & $1(2.9 \%)$ & 1 \\
\hline Auto-agglutinated & 0 & 0 & 0 & $1(2.5 \%)$ & $4(10.2 \%)$ & 0 & 4 \\
\hline $\begin{array}{l}\text { Total number of identified } \\
\text { serotypes/medium }\end{array}$ & 7 & 7 & 6 & 8 & 8 & 6 & \\
\hline
\end{tabular}

MSRV, modified semi-solid Rappaport-Vassiliadis medium; DIASALM, diagnostic semi-solid Salmonella medium; SMS, Simple Method Salmonella; RV, Rappaport Vassiliadis; RVS, Rappaport Vassiliadis broth with Soya; MKTTn, Muller Kauffmann Tetrathionate novobiocin broth. 
Calculation of the kappa value reveals an almost perfect agreement between MSRV and DIASALM $(\kappa=0.95)$, between MSRV and SMS $(\kappa=0.95)$, and between DIASALM and SMS $(\kappa=0.94)$. Between all the other media, a substantial $(\kappa=0.61-$ 0.80 ) agreement was found (Table 2).

The combined use of MSRV with RVS, MSRV with MKTTn, or DIASALM with RVS increased the detection rate to 63 (88.7\%) positive samples. Combining MSRV or DIASALM with RVS and MKTTn led to a rate of $94.4 \%$ (67/71 positive duodenal samples).

In total, 113 isolates were collected from the MSRV media, of which 57 were picked close to the inoculation drop and 56 at the edge of the migration zone. On DIASALM and SMS, 51 and 50 isolates, respectively, were picked close to the drop and 55 and 49 , respectively, at the edge of the migration zone.

From each slaughterhouse, 4-29 pigs tested positive, and Salmonella was isolated in the duodenal content of pigs originating from $28(50 \%)$ slaughter batches. For each batch, an average of 6.5 pigs were sampled, with 2.5 of these (on average) being Salmonella positive.

\section{Evaluation of strain motility}

In four of the nine pigs where Salmonella was only isolated after enrichment in one of the broths, serotyping of the isolates $(n=9)$ was not possible due to auto-agglutination (Table 3). Six of the nine isolates were able to migrate in MSRV when inoculated at the concentration level of $10^{6} \mathrm{cfu} / \mathrm{mL}$. When inoculated at the lower concentration level $\left(10^{3} \mathrm{cfu} / \mathrm{mL}\right)$, only two did migrate on MSRV. In five pigs, Salmonella could only be isolated using RV and MKTTn $(n=9)$, and different serotypes were identified (Typhimurium [n=3], Arizonae [ $n=3]$, Derby [ $n=2]$, and O4:i:- $[n=1])$. Eight of the nine isolates were able to migrate on MSRV when inoculated at the concentration level of $10^{6} \mathrm{cfu} / \mathrm{mL}$. When inoculated at the lower concentration level $\left(10^{3} \mathrm{cfu} / \mathrm{mL}\right)$, this number was declined to three. One of the three Salmonella Typhimurium isolates showed no migration capacity when inoculated at both concentration levels.

The 31 isolates selected from duodenal samples where Salmonella could be isolated from all the enrichment media showed full migration capacity on MSRV when inoculated at both concentration levels.

\section{Salmonella serotyping}

In total, 595 isolates from the duodenal content of 71 pigs were further serotyped. Table 3 shows the number of pigs for which a particular serotype had been isolated by the different enrichment media used. In general, Salmonella Typhimurium was less isolated after enrichment in RVS and Salmonella Derby after enrichment in RV. Salmonella Anatum was equally detected in all types of enrichment media used, but was in terms of percentage more identified after enrichment in the different broths. Salmonella Brandenburg and Salmonella Rissen were not isolated after enrichment in MKTTn, while Salmonella O4:i:- was not detected after enrichment in DIASALM and SMS. Further, it was not possible to isolate Salmonella Ohio from MSRV, SMS, and MKTTn. Salmonella Arizonae could only be detected in isolates of MKTTn (Table 3).

No Salmonella could be detected from the edge of the migration zone on SMS $(n=9)$, MSRV $(n=3)$, and DIASALM $(n=3)$ in nine animals, while Salmonella (Typhimurium, Derby, and Brandenburg) was isolated from cultures taken close

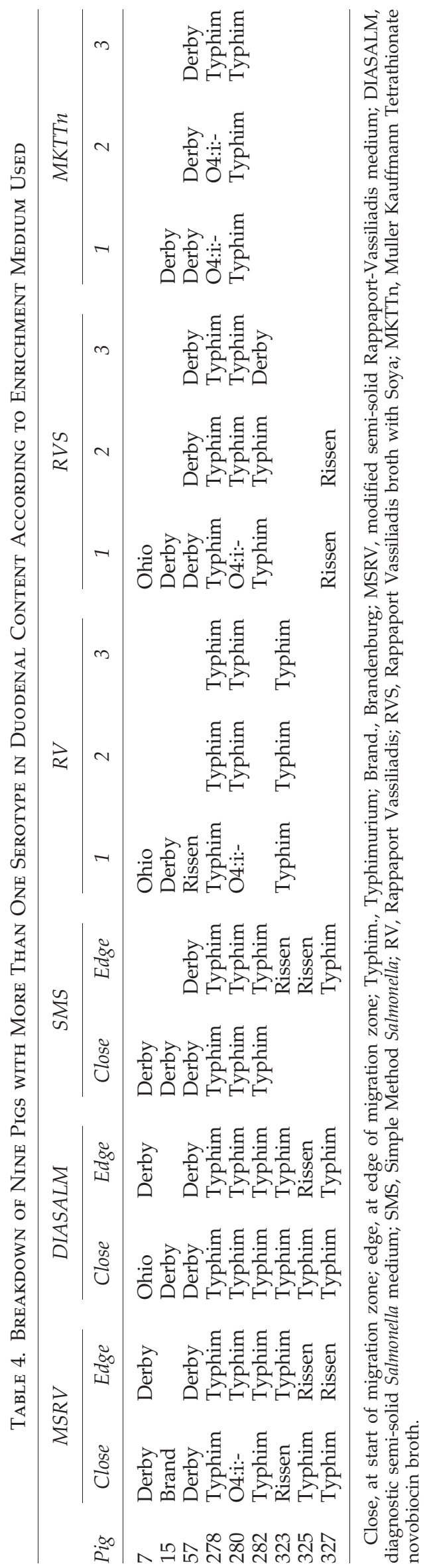


to the inoculation drop. In 15 pigs, Salmonella (Typhimurium, Derby, and Rissen) could be obtained from the edge of the migration zone, but not close to the inoculation drop (SMS $[n=8]$, DIASALM [ $n=7]$, and MSRV [ $n=2]$ ).

The number of serotypes found in the isolates of a single slaughter batch varied from one to three. In the majority of the positive pigs $(86.6 \%)$, only one serotype was present, and in the remaining pigs, two serotypes were present (Table 4). Differences in serotypes amongst all the isolates were found between and within the media used.

\section{Salmonella genotyping}

Genotyping was performed on 578 isolates, resulting in 34 different genotypes. For Salmonella Typhimurium, 19 genotypes were obtained, for Salmonella Derby five, for Salmonella O4:i:- three, for Salmonella Rissen and Salmonella Brandenburg two, and for the remaining serotypes one. In MSRV, $82.3 \%$ of the genotypes were identified; in DIASALM 73.5\%; in SMS $64.7 \%$; and in the liquid media RV, RVS, and MKTTn, 58.8\%, $44.1 \%$, and $50.0 \%$, respectively. Different genotypes (151 isolates) within and/or between the media were found in the duodenal content of $15(24.2 \%)$ pigs. For seven pigs, the difference in Salmonella strain could be explained by a difference in serotype, while for the remaining eight pigs, different genotypes were found within the same serotype. The different genotypes were detected between the different media and within the same medium (Table 5).

The number of genotypes found in the duodenal content of pigs per slaughter batch varied from one to six. The number of different genotypes in each pig varied from one to four, with one genotype found in $77.6 \%$ of the pigs, two genotypes in $16.4 \%$, three in $4.5 \%$, and four in $1.5 \%$.

\section{Discussion}

The detection rate of Salmonella isolated from naturally contaminated pig samples depends on the enrichment medium used. This study showed that, although semi-solid media are most suitable as enrichment media, a combination of different media is necessary to increase the relative sensitivity. Migration capacity can be influenced by the Salmonella serotype (auto-agglutinated or not) and depends on the concentration level of the organism in the sample. Isolates originating from liquid media only were in $77.8 \%$ of cases able to migrate on MSRV when inoculated from a high concentration. At a lower concentration $\left(10^{3} \mathrm{cfu} / \mathrm{mL}\right)$, migration capacity decreased to $27.8 \%$. If Salmonella is present in the pre-enrichment broth (BPW) at low concentrations, it can easily be missed when semi-solid media are used in the isolation protocol (ISO, 2007). In our procedure, the combination of MSRV or DIASALM with either RVS or MKTTn increased the relative sensitivity from $83.1 \%$ (MSRV) or $81.7 \%$ (DIASALM) up to $88.7 \%$. These results are in accordance with those obtained by Voogt et al. (2001), Dam-Deisz et al. (2003), and Botteldoorn et al. (2003), indicating that combining media (MSRV or DIASALM with RV) yields a higher number of Salmonellapositive samples. The highest relative sensitivity (94.4\%) in this study was achieved only by combining three different enrichment media (one semi-solid and two liquid media), increasing the labor intensity and therefore the costs involved.

The distribution of the serotypes showed that some serotypes were less or not recovered from certain media. This finding is also reported by Dam-Deisz et al. (2003), although the patterns of serotype detection in the different media used by these authors (MSRV, DIASALM, and RV) are not similar to those found in the present study. Future experimental studies with different serotypes in porcine fecal samples will allow us to gain more insight into the behavior of Salmonella serotypes in the standard MSRV medium and also in other enrichment media. Singer et al. (2009) showed already that the probability of detecting a specific Salmonella serotype in a sample depends on its ability to compete in the cultivation media and on the specific mixture of Salmonella bacteria present in the sample. However, explanations for this variability within serotypes need to be further examined.

More than one serotype was identified in the duodenal content of $13.4 \%$ of the pigs. The isolation of multiple serotypes from individual pigs has previously been reported (Kampelmacher et al., 1962; O'Carroll et al., 1999; Funk et al., 2000) and is important regarding epidemiological studies. Rostagno et al. (2005) demonstrated that asynchronous growth curves among serotypes were due to the selective enrichment media used in the Salmonella isolation protocol. In our study, differences in serotypes were predominantly found within the same media, demonstrating that it is useful to select more than one colony per sample. For semi-solid media, samples should be taken both close to the inoculation drop and also on the edge of the migration zone. In this way, less motile Salmonella serotypes

Table 5. Breakdown of Eight Pigs with More Than One Genotype Within Same Serotype According to Enrichment Medium Used

\begin{tabular}{|c|c|c|c|c|c|c|c|c|c|c|c|c|c|c|c|c|c|}
\hline \multirow[b]{2}{*}{ SH } & \multirow[b]{2}{*}{ Batch } & \multirow[b]{2}{*}{ Pig } & \multicolumn{2}{|c|}{$M S R V$} & \multicolumn{2}{|c|}{ DIASALM } & \multicolumn{2}{|c|}{ SMS } & \multicolumn{3}{|c|}{$R V$} & \multicolumn{3}{|c|}{$R V S$} & \multicolumn{3}{|c|}{ MKTTn } \\
\hline & & & Close & Edge & Close & Edge & Close & Edge & 1 & 2 & 3 & 1 & 2 & 3 & 1 & 2 & 3 \\
\hline \multirow[t]{5}{*}{ B } & 9 & 139 & $\mathrm{~T}_{19}$ & $\mathrm{~T}_{19}$ & $\mathrm{~T}_{19}$ & $\mathrm{~T}_{9}$ & $\mathrm{~T}_{19}$ & $\mathrm{~T}_{19}$ & $\mathrm{~T}_{19}$ & $\mathrm{~T}_{19}$ & $\mathrm{~T}_{9}$ & $\mathrm{~T}_{19}$ & $\mathrm{~T}_{19}$ & $\mathrm{~T}_{19}$ & $\mathrm{~T}_{19}$ & $\mathrm{~T}_{19}$ & \\
\hline & & 142 & $\mathrm{~T}_{9}$ & $\mathrm{~T}_{13}$ & $\mathrm{~T}_{15}$ & $\mathrm{~T}_{15}$ & $\mathrm{~T}_{15}$ & $\mathrm{~T}_{15}$ & $\mathrm{~T}_{15}$ & $\mathrm{~T}_{15}$ & & $\mathrm{~T}_{15}$ & $\mathrm{~T}_{15}$ & & $\mathrm{~T}_{15}$ & $\mathrm{~T}_{15}$ & \\
\hline & 15 & 273 & $\mathrm{~T}_{13}$ & $\mathrm{~T}_{13}$ & $\mathrm{~T}_{13}$ & $\mathrm{~T}_{13}$ & $\mathrm{~T}_{16}$ & $\mathrm{~T}_{13}$ & $\mathrm{~T}_{13}$ & $\mathrm{~T}_{13}$ & $\mathrm{~T}_{13}$ & $\mathrm{~T}_{13}$ & $\mathrm{~T}_{13}$ & $\mathrm{~T}_{13}$ & $\mathrm{~T}_{13}$ & $\mathrm{~T}_{13}$ & $\mathrm{~T}_{13}$ \\
\hline & & 280 & O4:i:- ${ }_{1}$ & $\mathrm{~T}_{13}$ & $\mathrm{~T}_{13}$ & $\mathrm{~T}_{13}$ & $\mathrm{~T}_{13}$ & $\mathrm{~T}_{13}$ & O4:i:- ${ }_{1}$ & $\mathrm{~T}_{13}$ & $\mathrm{~T}_{13}$ & O4:i:-33 & $\mathrm{T}_{13}$ & $\mathrm{~T}_{13}$ & $\mathrm{~T}_{13}$ & $\mathrm{~T}_{13}$ & $\mathrm{~T}_{13}$ \\
\hline & 16 & 314 & $\mathrm{~T}_{14}$ & $\mathrm{~T}_{12}$ & $\mathrm{~T}_{12}$ & $\mathrm{~T}_{12}$ & $\mathrm{~T}_{14}$ & & & & & & & & $\mathrm{~T}_{12}$ & & \\
\hline \multirow[t]{2}{*}{ D } & 22 & 325 & $\mathrm{~T}_{6}$ & $\mathrm{R}_{2}$ & $\mathrm{~T}_{7}$ & $\mathrm{R}_{1}$ & & $\mathrm{R}_{1}$ & & & & & & & & & \\
\hline & 24 & 353 & $\mathrm{~T}_{4}$ & $\mathrm{~T}_{4}$ & & $\mathrm{~T}_{4}$ & & & $\mathrm{~T}_{4}$ & $\mathrm{~T}_{2}$ & $\mathrm{~T}_{4}$ & & & & & & \\
\hline $\mathrm{F}$ & 28 & 456 & $\mathrm{~T}_{14}$ & $\mathrm{~T}_{11}$ & $\mathrm{~T}_{11}$ & $\mathrm{~T}_{14}$ & $\mathrm{~T}_{1}$ & $\mathrm{~T}_{1}$ & $\mathrm{~T}_{14}^{4}$ & $\mathrm{~T}_{1}$ & $\mathrm{~T}_{1}$ & & & & $\mathrm{~T}_{1}$ & $\mathrm{~T}_{1}$ & $\mathrm{~T}_{1}$ \\
\hline
\end{tabular}

$\mathrm{SH}$, slaughterhouse; Batch, slaughter batch; T, Typhimurium; R, Rissen; close, at start of migration zone; edge, on edge of migration zone; MSRV, modified semi-solid Rappaport-Vassiliadis medium; DIASALM, diagnostic semi-solid Salmonella medium; SMS, Simple Method Salmonella; RV, Rappaport Vassiliadis; RVS, Rappaport Vassiliadis broth with Soya; MKTTn, Muller Kauffmann Tetrathionate novobiocin broth. 
can also be detected. An explanation for the decreased motility of some strains on MSRV cannot be given by the results of this study, as all genotypes (except one) which were found at the start of the migration zone but not on the edge migrated in samples belonging to other pigs. It is possible that the salmonellae were overgrown by competitive bacteria present in the duodenal content of the pig, hindering isolation. In total, 34 strains were found, with the highest diversity within the serotype Typhimurium. The diverse nature of Salmonella Typhimurium has also been demonstrated by De Busser et al. (2011), showing that in 26 Salmonella Typhimurium isolates obtained from the duodenal content of naturally infected slaughtered pigs, six different genotypes were identified. The same study showed that in a total of 162 Salmonella Typhimurium isolates obtained from slaughterhouse samples, 32 different genotypes could be detected. Although Salmonella Typhimurium has often been considered as very clonal (On and Baggesen, 1997; Gebreyes, 2006), our results illustrate that even without additional phenotypic characterization a wide variety of genotypes belonging to Salmonella Typhimurium could be obtained. In $13 \%$ of the duodenal content of positive pigs, different strains were found within the same serotype. These differences were predominantly seen within the same medium. This finding again emphasizes the fact that examining more parts of the migration zone of semi-solid media or colonies derived from the enrichment broths increases the probability of detecting multiple genotypes.

\section{Conclusion}

This study shows that even by sampling a rather low number of pigs, multiple sero- and genotypes can be detected within a slaughter batch as well as within one pig. Taking into account the variation resulting from the type of enrichment medium used and the number of colonies taken, it is clear that obtaining realistic and valid data is a real challenge. Although increasing the number of colonies analyzed and using multiple enrichment media inherently involve higher costs and more labor, on occasions when detecting the Salmonella source is of primary importance, such as in outbreak investigations, it should be seriously considered.

\section{Disclosure Statement}

No competing financial interests exist.

\section{References}

Botteldoorn N, Heyndrickx M, Rijpens N, Grijspeerdt K, Herman L. Salmonella on pig carcasses: Positive pigs and cross contamination in the slaughterhouse. J Appl Microbiol 2003; 95:891-903.

Dam-Deisz WDC, Maas HME, Nagelkerke N, van de Giessen AW. Vergelijking van selectieve media voor de isolatie van salmonella spp. uit fecesmonsters van vleesvarkens, vleeskalveren en melkkoeien [Comparison of selective enrichment media for the isolation of Salmonella spp. from fecal samples from fattening pigs, veal calves and dairy cows]. De Ware(n) Chemicus 2003;3:143-151. (In Dutch.)

De Busser EV, Maes D, Houf K, Dewulf J, Imberechts H, Bertrand S, De Zutter L. Detection and characterization of Salmonella in lairage, on pig carcasses and intestines in five slaughterhouses. Int J Food Microbiol 2011;145:279-286.
[EFSA] European Food Safety Authority. Report of the Task Force on Zoonoses Data Collection on the Analysis of the baseline survey on the prevalence of Salmonella in slaughter pigs, in the EU, 2006-2007 [1]—Part A: Salmonella prevalence estimates. EFSA J 2008;135:1-111. Available at: http://www .efsa.europa.eu/en/efsajournal/pub/135r.htm, accessed December 1, 2012.

[EFSA] European Food Safety Authority. Scientific opinion on a quantitative microbiological risk assessment of Salmonella in slaughter and breeder pigs. EFSA J 2010a;8:1547. Available at: http://www.efsa.europa.eu/en/efsajournal/pub/1547.htm, accessed December 1, 2012.

[EFSA] European Food Safety Authority. Scientific opinion on monitoring and assessment of the public health risk of "Salmonella Typhimurium-like" strains. EFSA J 2010b;8:1826. Available at: http://www.efsa.europa.eu/en/efsajournal/ pub/1826.htm, accessed December 1, 2012.

Funk JA, Davies PR, Nichols MA. The effect of fecal sample weight on detection of Salmonella enterica in swine feces. J Vet Diagn Invest 2000;12:412-418.

Gebreyes WA, Altier C, Thakur S. Molecular epidemiology and diversity of Salmonella serovar Typhimurium in pigs using phenotypic and genotypic approaches. Epidemiol Infect 2006;134:187-198.

Grimont PAD, Grimont F, Bouvet P. Taxonomy of the genus Salmonella. In: Salmonella in Domestic Animals. Wray C, Wray A (eds.). Wallingford, Oxon, UK: CABI Publishing, 2000, p. 4. Hald T, Wingstrand A, Swanenburg M, von Altrock A, Thorberg BM. The occurence and epidemiology of Salmonella in European pig slaughterhouses. Epidemiol Infect 2003;131:1187-1203.

Harvey RW, Price TH. Isolation of salmonellas from sewagepolluted river water using selenite $\mathrm{F}$ and Muller-Kauffmann tetrathionate. J Hyg (Lond) 1976;77:333-339.

ISO 6579:2002/Amd 1 2007. Amendment 1 Annex D: Detection of Salmonella spp. in animal feces and in environmental samples from the primary production stage.

Kampelmacher EH, Guinee PAM, Van Keulen A. Further studies on Salmonella in slaughterhouses and in normal slaughter pigs. J Vet Med B 1962;10:1-27.

Landis JR, Koch GG. The measurement of observer agreement for categorical data. Biometrics 1977;33:159-174.

May HG, Goodner K. Cultural and antigenic studies on Salmonella gallinarum and Salmonella pullorum. J Bacteriol 1927; 13:129-146.

O'Carroll JM, Davies PR, Correa MT. Effect of sample storage and delayed secondary enrichment on detection of Salmonella spp. in swine feces. Am J Vet Res 1999;60:359-362.

O'Donoghue D, Winn E. Comparison of the MSRV method with an in-house conventional method for the detection of Salmonella in various high and low moisture foods. Lett Appl Microbiol 1993;17:174-177.

On SLW, Baggesen DL. Determination of clonal relationships of Salmonella Typhimurium by numerical analysis of macrorestriction profiles. J Appl Microbiol 1997;83:699-706.

Popoff MY, Le Minor L. Antigenic Formulas of the Salmonella Serovars. Paris: WHO Collaborating Centre for Reference and Research on Salmonella, Institute Pasteur, 1992.

Rasschaert G, Houf K, Imberechts H, Grijspeerdt K, De Zutter L, Heyndrickx M. Comparison of five repetitive-sequence-based PCR typing methods for molecular discrimination of Salmonella enterica isolates. J Clin Microbiol 2005;43:3615-3623.

Read SC, Irwin RJ, Poppe C, Harris J. A comparison of two methods for isolation of Salmonella from poultry litter samples. Poult Sci 1994;73:1617-1621. 
Ribot EM, Fair MA, Gautom R, Cameron DN, Hunter SB, Swaminathan B, Barrett TJ. Standardization of pulsed-field gel electrophoresis protocols for the subtyping of Escherichia coli O157:H7, Salmonella, and Shigella for PulseNet. Foodborne Pathog Dis 2006;3:59-67.

Rostagno MH, Gailey JK, Hurd HS, McKean JD, Leite RC. Culture methods differ on the isolation of Salmonella enterica serotypes from naturally contaminated swine fecal samples. J Vet Diagn Invest 2005;17:80-83.

Singer RS, Mayer AE, Hanson TE, Isaacson RE. Do microbial interactions and cultivation media decrease the accuracy of Salmonella surveillance systems and outbreak investigations? J Food Prot 2009;72:707-713.
Voogt N, Raes M, Wannet WJB, Henken AM, van de Giessen AW. Comparison of selective enrichment media for the detection of Salmonella in poultry feces. Lett Appl Microbiol 2001;32:89-92.

Address correspondence to: Emily V. De Busser, DVM, PhD Faculty of Veterinary Medicine Ghent University Salisburylaan 133 B-9820 Merelbeke, Belgium

E-mail: Emily.DeBusser@Ugent.be 